\title{
Early Experience and the Feasibility of the Firefly Technology for Central Lymph Node Dissection (CLND) in Robotic Thyroidectomy Using the BABA
}

\author{
Jin Ah Choi, M.D., Jeeyeon Lee, M.D., Jin Hyang Jung, M.D., Ho Yong Park, M.D., Wan Wook Kim, M.D.
}

Department of Surgery, School of Medicine, Kyungpook National University, Daegu, Korea

\begin{abstract}
In the bilateral axillo-breast approach (BABA), the camera is inserted through the areolar incision, and this raises the concern it might be difficult to identify the lymph nodes (LN). The purpose of this study is to evaluate the feasibility of the Firefly for central lymph node dissection (CLND) in robotic thyroidectomy using the BABA. This study evaluated 18 patients who underwent robotic surgery using Firefly between December 2015 and March 2016. For LN mapping, $0.05 \mathrm{ml}$ of ICG was injected into the thyroid 3 4 minutes before CLND. Green-stained LN could be detected easily through a near-infrared camera. The number of retrieved LNs was 7.8 \pm 3.0 after CLND using the Firefly, which was higher than the $6.7 \pm 0.2$ reported in previous surgeries. In addition, it helped to distinguish between the parathyroid and the LNs. The Firefly technology was helpful in identifying the LNs, guiding the CLND and performing a complete CLND.
\end{abstract}

Keywords: Bilateral axillo-breast approach, Fluorescence image, Indocyanine green, Node dissection, Robot

Supplementary video file: This article contains supplementary material (https://doi.org/10.7602/ jmis.2017.20.3.117).

This is an Open Access article distributed under the terms of the Creative Commons Attribution Non-Commercial License (http:// creativecommons.org/licenses/by-nc/4.0/) which permits unrestricted non-commercial use, distribution, and reproduction in any medium, provided the original work is properly cited.
Received June 27, 2017

Revised 1st August 1, 2017

2nd August 16, 2017

Accepted August 16, 2017

Corresponding author

Wan Wook Kim

Department of Surgery, School of

Medicine, Kyungpook National

University, 807 Hogukno, Buk-gu,

Daegu 41404, Korea

Tel: +82-53-200-2705

Fax: +82-53-200-2027

E-mail:kww1324@naver.com

\section{INTRODUCTION}

Robotic surgery using the bilateral axillo-breast approach (BABA) offers an excellent cosmetic result without neck scar. However, because the camera is inserted downward through the breast areola incision, the deep central lymph nodes are difficult to find and easy to miss. Thus, there was the concern that central lymph node dissection (CLND) using this approach was incomplete, compared to open surgery. ${ }^{1}$ Recently, Firefly technology using fluorescence imaging (FI) was developed with the da Vinci ${ }^{\circledR}$ Surgical system (Intuitive Surgical Inc., Sunnyvale, CA, USA) and applied in some cancer sur- geries. ${ }^{2}$ The purpose of this study is to report an early experience and the feasibility of the Firefly technology for CLND in robotic thyroidectomy using the BABA.

\section{METHODS}

This study evaluated 18 patients who underwent robotic surgery with CLND using FI for papillary thyroid carcinoma between December 2015 and March 2016 (Table 1). Intraoperatively, $0.05 \mathrm{ml}$ of indocyanine green (ICG) was injected into the thyroid parenchyma under the view of a robotic camera, immediately prior to CLND (Fig. 1. The assistant inserts a 
Table 1. Clinicopathologic characteristics

\begin{tabular}{|c|c|c|c|c|c|c|c|}
\hline & Age/Sex & Operation & $\begin{array}{l}\text { Tumor } \\
\text { size }(\mathrm{cm})\end{array}$ & $\begin{array}{c}\text { Retrieved } \\
\text { LN (n) }\end{array}$ & $\begin{array}{c}\text { Metastatic } \\
\text { LN (n) }\end{array}$ & $\begin{array}{c}\text { РTH } \\
\text { (pg/ml) }\end{array}$ & Complication \\
\hline 1 & $54 / F$ & $\mathrm{RL}+$ ipsilateral CLND & 0.2 & 12 & 0 & 44.0 & Non \\
\hline 2 & $47 / F$ & RL+ipsilateral CLND, contralateral LN picking & 0.6 & 9 & 0 & 54.4 & Non \\
\hline 3 & $40 / F$ & TT + bilateral CLND & 0.8 & 11 & 4 & 44.3 & Non \\
\hline 4 & $51 / F$ & LL+ipsilateral CLND & 0.3 & 5 & 0 & 65.7 & Non \\
\hline 5 & $39 / F$ & LL+ipsilateral CLND & 0.3 & 6 & 0 & 26.4 & Non \\
\hline 6 & $51 / \mathrm{F}$ & TT+ipsilateral CLND & 0.4 & 8 & 1 & 36.7 & Non \\
\hline 7 & $33 / \mathrm{F}$ & TT+bilateral CLND & 2.3 & 12 & 2 & 2.5 & Transient hypocalcemia \\
\hline 8 & $43 / \mathrm{F}$ & TT+ipsilateral CLND & 0.5 & 3 & 0 & 31.2 & Non \\
\hline 9 & $46 / F$ & LL+ ipsilateral CLND & 1.0 & 4 & 1 & 81.1 & Non \\
\hline 10 & $47 / F$ & TT+bilateral CLND & 0.8 & 9 & 3 & 3.1 & Transient hypocalcemia \\
\hline 11 & $55 / F$ & RL+ipsilateral CLND & 0.6 & 4 & 0 & 36.5 & Non \\
\hline 12 & $43 / \mathrm{F}$ & LL+ipsilateral CLND & 0.3 & 1 & 0 & 62.5 & Non \\
\hline 13 & $40 / F$ & RL+ipsilateral CLND & 1.8 & 15 & 0 & 43 & Non \\
\hline 14 & $27 / F$ & TT+ipsilateral CLND & 0.7 & 6 & 2 & 5 & Transient hypocalcemia \\
\hline 15 & $38 / F$ & TT+ipsilateral CLND & 0.7 & 10 & 8 & 20 & Non \\
\hline 16 & $43 / \mathrm{F}$ & TT+ipsilateral CLND & 0.7 & 10 & 6 & 2.5 & Transient hypocalcemia \\
\hline 17 & $41 / F$ & LL+ipsilateral CLND & 1.0 & 3 & 0 & 29.4 & Non \\
\hline 18 & $28 / F$ & TT+ipsilateral CLND & 0.5 & 12 & 1 & 30.2 & Non \\
\hline
\end{tabular}

$R L=$ Right lobectomy; $L L=$ Left lobectomy; $T T=$ Total thyroidectomy; CLND= central lymph node dissection; PTH $(\mathrm{pg} / \mathrm{mll}=$ immediate postoperative parathyroid hormone.

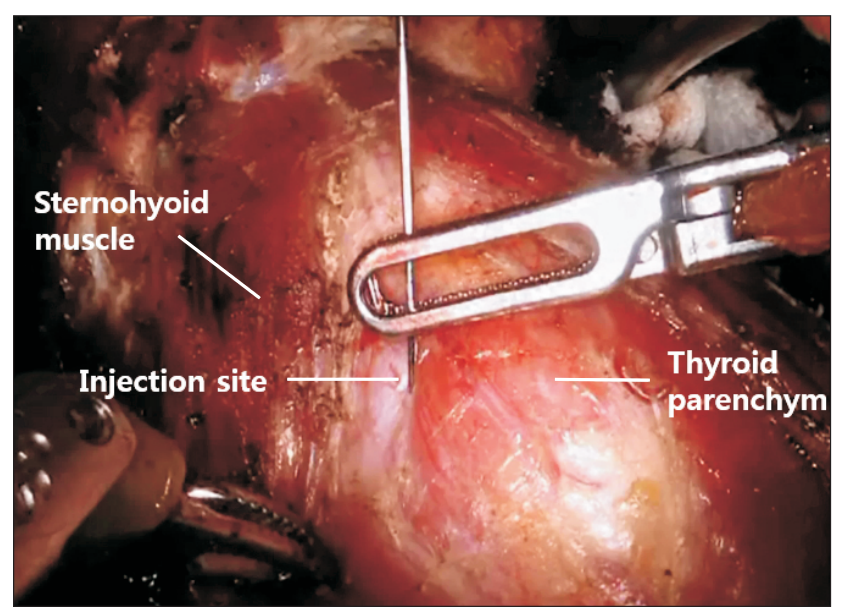

Fig. 1. The assistant inserts a spinal needle through the skin and slowly injects the ICG, and massages the thyroid gently.

spinal needle through the skin and slowly injects the ICG, and massages the thyroid gently). Initially, $0.1 \mathrm{ml}$ of ICG was injected, some of which spilled into the surgical site. The spill-

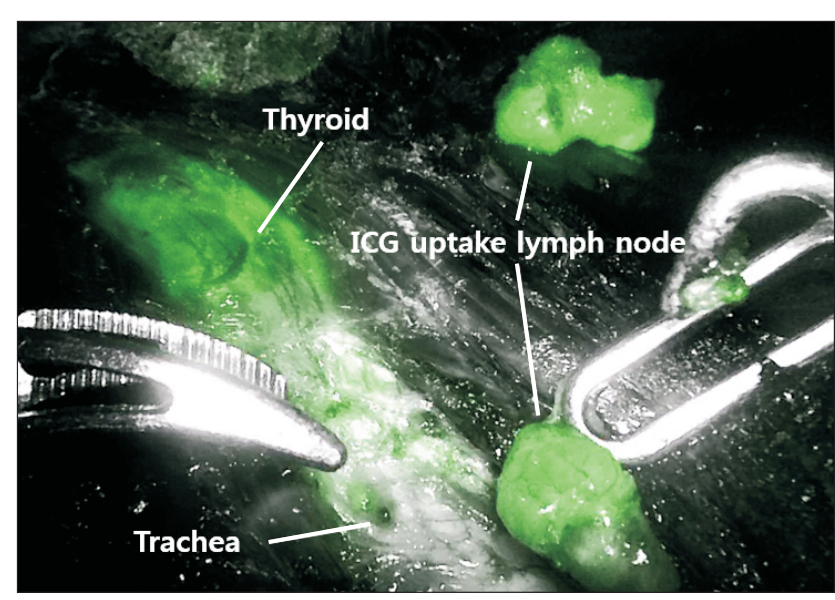

Fig. 2. The surgeon performs pretracheal lymph node dissection and can see the ICG uptake LNs using Firefly.

age interfered with the identification of lymph nodes (LNs); hence, the volume of ICG was reduced to $0.05 \mathrm{ml}$. To reduce spillage of the ICG, a $25-\mathrm{G}$ spinal needle was used for injec- 
tion.

After the injection, a piece of gauze was used to gently massage the thyroid parenchyma for $3 \sim 4$ min to facilitate the flow of ICG through the lymphatics. As ICG is visualized in green color through the Firefly system using near-infrared FI, a surgeon can easily visualize the uptake of ICG by the LNs in real time (Fig. 2. The surgeon performs pretracheal lymph node dissection and can see the ICG uptake LNs using Firefly). The surgery was completed after confirming no further uptake of ICG by the LNs in the operative bed.

\section{RESULTS}

In all the patients, uptake of ICG by the LNs, was detected after ICG injection. Although LNs could be identified grossly without FI, it was easier for the surgeon to identify them by mapping using FI. LN mapping was helpful to ensure completeness of the CLND, to reduce the chances of missing hidden LNs. The number of retrieved LNs was 7.8 23.0 in the operations using FI, which was higher than the $6.7 \pm 0.2$ reported in previous 300 surgeries without $\mathrm{FI}^{3}{ }^{3}$ In addition to identifying the LNs, FI was helpful to distinguish LN from the parathyroid gland, because the parathyroid gland does not show ICG uptake like LNs. In some cases, we could not only identify the ipsilateral LNs but also the contralateral LNs. Additional operation time was not necessary, and no adverse effects of ICG were observed.

\section{DISCUSSION}

In this study, the Firefly technology was applied for better LN identification through LN mapping and completeness of the CLND, compared to sentinel LN biopsy, which is used for LN staging and minimizing complications through a minimally invasive assessment. The possibility of missing the deeply located LNs seems to be overcome through FI in ro- botic thyroidectomy.

When ICG was intravenously injected, the parathyroid glands, being hypervascular, showed a relatively faster uptake than the thyroid gland; thus, staining occurred earlier in the parathyroid gland than the thyroid glands, which was useful for localization of the parathyroid glands in another study. ${ }^{4}$ In this study, however, ICG was injected into the thyroid parenchyma and reached the LNs through the lymphatics. Thus, ICG uptake by the parathyroid glands was relatively lower than that of the LN. The difference in relative uptake was useful in distinguishing between the parathyroid glands and LN, to save the parathyroid glands. To properly identify LNs with ICG uptake, a clean operative field should be maintained. Even though it is unclear how long LNs can maintain the ICG uptake, this study confirmed that the ICG uptake remained for 2 3 hours intraoperatively.

In conclusion, the Firefly technology using fluorescence imaging was helpful to identify LNs in real time and guide complete CLND in BABA robotic thyroidectomy.

\section{REFERENCES}

1) Kwak HY, Kim HY, Lee HY, et al. Robotic thyroidectomy using bilateral axillo-breast approach: Comparison of surgical results with open conventional thyroidectomy. J Surg Oncol 2015;111:141-145.

2) Herrera-Almario G, Patane M, Sarkaria I, Strong VE. Initial report of near-infrared fluorescence imaging as an intraoperative adjunct for lymph node harvesting during robot-assisted laparoscopic gastrectomy. J Surg Oncol 2016;113:768-770.

3) Kim WW, Jung JH, Park HY. A single surgeon's experience and surgical outcomes of 300 robotic thyroid surgeries using a bilateral axillo-breast approach. J Surg Oncol 2015;111:135-140.

4) Yu HW, Chung JW, Yi JW, et al. Intraoperative localization of the parathyroid glands with indocyanine green and Firefly(R) technology during BABA robotic thyroidectomy. Surg Endosc 2017;31:3020-3027. 